\title{
Usage of the homotopy analysis method for solving the nonlinear and linear integral equations of the second kind
}

\author{
Edyta Hetmaniok • Damian Słota • \\ Tomasz Trawiński • Roman Wituła
}

Received: 10 June 2013 / Accepted: 2 October 2013 / Published online: 24 November 2013 (C) The Author(s) 2013. This article is published with open access at Springerlink.com

\begin{abstract}
The paper presents an application of the homotopy analysis method for solving the nonlinear and linear integral equations of the second kind. In this method a series is created, sum of which (if the series is convergent) gives the solution of discussed equation. Conditions ensuring convergence of this series are presented in the paper. Error of approximate solution, obtained by considering only partial sum of the series, is also estimated. Examples illustrating usage of the investigated method are presented as well, including the example having practical application for calculating the charge in supply circuit of flash lamps used in cameras.
\end{abstract}

Keywords Homotopy analysis method · Nonlinear integral equation · Linear integral equation $\cdot$ Convergence $\cdot$ Error estimations

Mathematics Subject Classifications (2010) $65 \mathrm{R} 20 \cdot 45 \mathrm{G} 10 \cdot 45 \mathrm{~A} 05$

\section{Introduction}

Homotopy analysis method was developed by Shijun Liao [28-31, 33]. It enables to solve the operator equations of different kind. In particular, the method has found a number of applications in heat conduction problems [1, 16, 18, 26, 56]. It is also used, among others, for solving the nonlocal initial boundary value problem [35], nonlinear

\footnotetext{
E. Hetmaniok · D. Słota $(\bowtie) \cdot$ R. Wituła

Institute of Mathematics, Silesian University of Technology, Kaszubska 23, 44-100 Gliwice, Poland e-mail: damian.slota@polsl.pl

T. Trawiński

Mechatronics Division, Silesian University of Technology, Kaszubska 23, 44-100 Gliwice, Poland
} 
reaction-diffusion-convection problems [41] and fractional differential equations [4, $54,58]$. In several papers the method was used for solving the integro-differential equations [9, 14, 20, 43, 57]. Theoretical results concerning, among others, convergence of the method in case of differential equations are included in papers [29, 31, $38,39,44-46,52]$. Various modifications of homotopy analysis method have been also elaborated, for example, the spectral homotopy analysis method [37], the optimal homotopy analysis method [19] and the optimal homotopy asymptotic method [22, 34] (see also [33]).

In recent time there have appeared some papers describing application of the homotopy analysis method for solving integral equations. In papers [40, 42] the examples of using the method for solving the system of integral equations are presented. Whereas the examples of applying the method for solving the twodimensional integral equations can be found in works [5, 12]. Next, in papers [13, 36] the possibility of using the method for solving the fuzzy integral equations is shown. Paper [48] includes the examples of applying the discussed method for solving the Fredholm and Volterra integral equations of the first and second kind. Abbasbandy with the co-authors described the usage of homotopy analysis method for solving the nonlinear Fredholm and Volterra integral equations of the second kind $[2,53]$. In case of the Fredholm equation [2] it was proven that if the series obtained in result of homotopy analysis method is convergent then its sum satisfies the discussed equation. The same was proven for the Volterra equation as well [53]. Moreover, in case of this equation the uniqueness of solution was proven and the sufficient condition for convergence of created series was given. Additionally, it was proven in both papers that in the case of investigated integral equations the Adomian decomposition method represents a special case of the homotopy analysis method. Particular case of nonlinear equation (sought function is in power and inside of integral) is considered also in paper [10], in which the uniqueness of solution is proven together with the fact that sum of the series gives the sought solution.

Some modifications of homotopy analysis method have been also used for solving the integral equations. In particular, in paper [8] the discrete homotopy analysis method is applied which has been obtained by combining the homotopy analysis method with quadrature rules. Whereas, in paper [27] the multistage homotopy analysis method is used. In this method the interval, in which the considered equation is defined, is divided into several subintervals and next, in each one of them, the homotopy analysis method is applied.

In the current paper we intend to use the homotopy analysis method for solving the nonlinear and linear integral equations of the second kind. Discussed equations are in the more general form than the ones considered in [2, 10, 48, 53]. In particular, we prove in this paper that under appropriate assumptions the investigated equations possess unique solutions. We prove also that if the series, obtained in the course of using the method, is convergent then its sum is a solution of considered equation. Condition ensuring the series convergence is presented and the error of approximate solution, obtained by taking the partial sum of the series, is estimated. The paper includes also the example of using the homotopy analysis method for determining the approximate solution of the equation having some practical application for calculating the charge in supply circuit of flash lamps used in cameras. 


\section{Homotopy analysis method}

Homotopy analysis method serves for solving the operator equations

$$
N(u(x))=0, \quad x \in \Omega,
$$

where $N$ denotes the operator (in particular, it can be the nonlinear operator), whereas $u$ is the unknown function. In the first step of method we define the homotopy operator $\mathcal{H}$ in the following way

$$
\mathcal{H}(\Phi, p) \equiv(1-p) L\left(\Phi(x ; p)-u_{0}(x)\right)-p h N(\Phi(x ; p))
$$

where $p \in[0,1]$ is the embedding parameter, $h \neq 0$ denotes the convergence control parameter $[31,33,39,52], u_{0}$ describes the initial approximation of the solution of problem (1) and $L$ is the auxiliary linear operator with property $L(0)=0$.

Considering equation $\mathcal{H}(\Phi, p)=0$ we get the so-called zero-order deformation equation

$$
(1-p) L\left(\Phi(x ; p)-u_{0}(x)\right)=p h N(\Phi(x ; p)) .
$$

For $p=0$ we have $L\left(\Phi(x ; 0)-u_{0}(x)\right)=0$ which implies that $\Phi(x ; 0)=u_{0}(x)$. Whereas for $p=1$ we have $N(\Phi(x ; 1))=0$ which means that $\Phi(x ; 1)=u(x)$, where $u$ is the sought solution of (1). In this way, the change of parameter $p$ from zero to one corresponds with the change of problem from the trivial problem to the original one (and with the change of solution from $u_{0}$ to $u$ ).

By expanding function $\Phi(x ; p)$ into the Maclaurin series with respect to parameter $p$ we receive

$$
\Phi(x ; p)=\Phi(x ; 0)+\left.\sum_{m=1}^{\infty} \frac{1}{m !} \frac{\partial^{m} \Phi(x ; p)}{\partial p^{m}}\right|_{p=0} p^{m} .
$$

By designating

$$
u_{m}(x)=\left.\frac{1}{m !} \frac{\partial^{m} \Phi(x ; p)}{\partial p^{m}}\right|_{p=0}, \quad m=1,2,3, \ldots,
$$

the previous relation can be written in the following form

$$
\Phi(x ; p)=u_{0}(x)+\sum_{m=1}^{\infty} u_{m}(x) p^{m} .
$$

If the above series is convergent for $p=1$ then we get the sought solution

$$
u(x)=\sum_{m=0}^{\infty} u_{m}(x)
$$

In order to determine function $u_{m}$ we differentiate the left and the right side of relation (3) $m$ times with respect to parameter $p$, next we divide the received result by $m$ ! and we substitute $p=0$. In this way we obtain the so-called $m$ th-order deformation equation $(m>0)$ :

$$
L\left(u_{m}(x)-\chi_{m} u_{m-1}(x)\right)=h R_{m}\left(\bar{u}_{m-1}, x\right),
$$


where $\bar{u}_{m-1}=\left\{u_{0}(x), u_{1}(x), \ldots, u_{m-1}(x)\right\}$ and

$$
\chi_{m}= \begin{cases}0 & m \leq 1 \\ 1 & m>1\end{cases}
$$

and

$$
R_{m}\left(\bar{u}_{m-1}, x\right)=\left.\frac{1}{(m-1) !}\left(\frac{\partial^{m-1}}{\partial p^{m-1}} N\left(\sum_{i=0}^{\infty} u_{i}(x) p^{i}\right)\right)\right|_{p=0} .
$$

If we are not able to determine the sum of series in (7) then we can accept the partial sum of this series

$$
\widehat{u}_{n}(x)=\sum_{m=0}^{n} u_{m}(x)
$$

as the approximate solution of considered equation.

Appropriate selection of the convergence control parameter $h$ has a big influence on the convergence region of series (7) and on the convergence rate as well $[33,38$, 45]. One of the methods for selecting the value of convergence control parameter is the so-called $h$-curve. To obtain this curve we need to investigate the behavior of a certain quantity of the exact solution as a function of parameter $h$ [29, 46]. This method enables to determine the effective region of the convergence control parameter, however it does not give the possibility to determine the value ensuring the fastest convergence [33]. Another method is the so-called "optimization method" proposed in paper [55] (see also [6, 33]). In this method we define the squared residual of governing equation

$$
E_{n}(h)=\int_{\Omega}\left(N\left[\widehat{u}_{n}(x)\right]\right)^{2} d x .
$$

Optimal value of the convergence control parameter is obtained by finding minimum of this squared residual. Whereas the effective region of the convergence control parameter is defined as

$$
\mathbf{R}_{h}=\left\{h: \lim _{n \rightarrow \infty} E_{n}(h)=0\right\} .
$$

For speed up the calculations Liao [33] suggested to replace the integral in formula (12) by its approximate value obtained by applying the quadrature rules. In examples presented by Liao the received optimal values of the convergence control parameter differ not much from the values obtained by applying formula (12). The residual error method for obtaining the convergence control parameter $(h)$, shown in (12), has been applied recently to a number of problems for nonlinear ODEs and PDEs [3, 7, 11, $19,21,32,39,49-51]$.

\section{Nonlinear integral equation}

We consider equation of the form

$$
u(x)-\int_{f(x)}^{g(x)} K(x, t) R(u(t)) d t=F(x),
$$


where $x \in[a, b], R: C[a, b] \rightarrow C[a, b]$ is the nonlinear operator, $f, g \in C[a, b]$, $a \leq f(x) \leq g(x) \leq b, K \in C([a, b] \times[a, b])$ and $F \in C[a, b]$, whereas function $u$ is sought. We assume that $R$ is the operator satisfying the Lipschitz condition

$$
\left\|R\left(v_{1}\right)-R\left(v_{2}\right)\right\| \leq s\left\|v_{1}-v_{2}\right\| \quad \text { for every } \quad v_{1}, v_{2} \in C[a, b]
$$

and some $s>0$. As norm of the function we take the supremum norm

$$
\|v\|=\sup _{x \in \Omega}|v(x)|
$$

in particular

$$
\|K\|=\sup _{(x, t) \in[a, b] \times[a, b]}|K(x, t)| \quad \text { and } \quad\|F\|=\sup _{x \in[a, b]}|F(x)| .
$$

Special cases of the above equation are the Fredholm and Volterra integral equations of the second kind.

Operators $L$ and $N$ can be define in the following way

$$
L(v)=v, \quad N(v)=v(x)-F(x)-\int_{f(x)}^{g(x)} K(x, t) R(v(t)) d t .
$$

Let $u_{0} \in C[a, b]$. In this case, by applying the homotopy analysis method we get the following formula for functions $u_{m}$ :

$$
u_{m}(x)=\chi_{m} u_{m-1}(x)+h R_{m}\left(\bar{u}_{m-1}, x\right),
$$

where $\chi_{m}$ and $R_{m}$ are defined by relations (9) and (10), respectively. By using definitions of the respective operators we obtain

$$
u_{1}(x)=h\left(u_{0}(x)-F(x)-\int_{f(x)}^{g(x)} K(x, t) R\left(u_{0}(t)\right) d t\right),
$$

and for $m \geq 2$ :

$$
\begin{aligned}
u_{m}(x)= & (1+h) u_{m-1}(x) \\
& -\frac{h}{(m-1) !} \int_{f(x)}^{g(x)} K(x, t)\left(\frac{\partial^{m-1}}{\partial p^{m-1}} R\left(\sum_{i=0}^{\infty} u_{i}(t) p^{i}\right)\right)_{p=0} d t
\end{aligned}
$$

In literature one can find the expression $\frac{\partial^{m-1}}{\partial p^{m-1}} R\left(\sum_{i=0}^{\infty} u_{i}(x) p^{i}\right)_{p=0}$ calculated for various nonlinear operators $R$. Most of these results are collected in monograph [33].

Now we proceed to prove that under appropriate assumptions (14) possesses a unique solution.

Theorem 1 If the following condition is fulfilled

$$
s\|K\|(b-a)<1,
$$

then (14) possesses at most one solution. 
Proof Let us suppose that there exist two solutions $u_{1}$ and $u_{2}$. Thus we have

$$
\begin{aligned}
\left\|u_{1}-u_{2}\right\| & =\left\|\int_{f(x)}^{g(x)} K(x, t)\left(R\left(u_{1}(t)\right)-R\left(u_{2}(t)\right)\right) d t\right\| \\
& \leq\|K\| \int_{f(x)}^{g(x)}\left\|R\left(u_{1}\right)-R\left(u_{2}\right)\right\| d t \leq s\|K\|(b-a)\left\|u_{1}-u_{2}\right\| .
\end{aligned}
$$

Hence we get

$$
(1-s\|K\|(b-a))\left\|u_{1}-u_{2}\right\| \leq 0 .
$$

So, if condition (19) is satisfied then equality $u_{1}=u_{2}$ must hold true.

We proceed now to prove the theorem ensuring that the sum of determined series is the solution of discussed equation.

Theorem 2 Let functions $u_{m}, m \geq 1$, be defined by relations (17) and (18). Then, if $s<1$ and series in (7) is convergent, the sum of this series is the solution of (14).

Proof Let series (7) be convergent. From the necessary condition for the series convergence we get that for any $x \in[a, b]$ :

$$
\lim _{m \rightarrow \infty} u_{m}(x)=0 \text {. }
$$

Let us designate

$$
H_{m}(x)=\left.\frac{1}{m !}\left(\frac{\partial^{m}}{\partial p^{m}} R\left(\sum_{i=0}^{+\infty} u_{i}(x) p^{i}\right)\right)\right|_{p=0} .
$$

If $R$ is the contraction mapping $(s<1)$ and series (7) converges to $u(x)$ then series $\sum_{m=0}^{\infty} H_{m}(x)$ converges to $R(u(x))$ (see [17]).

By using definition of operator $L$ we can write

$$
\begin{aligned}
\sum_{m=1}^{n} L\left(u_{m}(x)-\chi_{m} u_{m-1}(x)\right)= & \sum_{m=1}^{n}\left(u_{m}(x)-\chi_{m} u_{m-1}(x)\right) \\
= & u_{1}(x)+\left(u_{2}(x)-u_{1}(x)\right)+\left(u_{3}(x)-u_{2}(x)\right)+\ldots \\
& +\left(u_{n}(x)-u_{n-1}(x)\right)=u_{n}(x) .
\end{aligned}
$$

Hence

$$
\sum_{m=1}^{\infty} L\left(u_{m}(x)-\chi_{m} u_{m-1}(x)\right)=\lim _{n \rightarrow \infty} u_{n}(x)=0 .
$$

From (8) we receive

$$
h \sum_{m=1}^{\infty} R_{m}\left(\bar{u}_{m-1}, x\right)=\sum_{m=1}^{\infty} L\left(u_{m}(x)-\chi_{m} u_{m-1}(x)\right),
$$


that is, since $h \neq 0$ thus we have

$$
\sum_{m=1}^{\infty} R_{m}\left(\bar{u}_{m-1}, x\right)=0 .
$$

In result of some transformations we get successively

$$
\left.\begin{array}{rl}
0 & =\sum_{m=1}^{\infty} R_{m}\left(\bar{u}_{m-1}, x\right) \\
& =\sum_{m=1}^{\infty}\left(\frac { 1 } { ( m - 1 ) ! } \frac { \partial ^ { m - 1 } } { \partial p ^ { m - 1 } } \left[\sum_{i=1}^{\infty} u_{i}(x) p^{i}-F(x)\right.\right. \\
& \left.\left.-\int_{f(x)}^{g(x)} K(x, t) R\left(\sum_{i=1}^{\infty} u_{i}(t) p^{i}\right) d t\right]_{p=0}\right) \\
& =\sum_{m=1}^{\infty}\left(u_{m-1}(x)-\frac{1-\chi_{m}}{(m-1) !} F(x)\right. \\
& =\sum_{m=1}^{\infty}\left(u_{m-1}^{g(x)} K(x)-\frac{1-\chi_{m}}{(m-1) !} F(x)-\int_{f(x)}^{g(x)} K(x, t) H_{m-1}(t) d t\right) \\
& =\sum_{m=1}^{\infty} u_{m-1}(x)-F(x)-\int_{f(x)}^{g(x)} K(x, t) \sum_{m=1}^{\infty} H_{m-1}(t) d t \\
& =u(x)-F(x)-\int_{f(x)}^{g(x)} K(x, t) R(u(t)) d t .
\end{array}\right)
$$

Remark 1 In the above proof we used the fact that $R$ is the contraction mapping in order to ensure the convergence of series $\sum_{m=0}^{\infty} H_{m}(x)$ to $R(u(x))$, in case when series (7) converges to $u(x)$. The same convergence can be obtained under another assumptions, for example, when $R$ belongs to class $C^{\infty}$.

Now we present the sufficient condition for convergence of considered series.

Theorem 3 If parameter $h$ is selected in such a way that the constants $\beta_{h} \in(0,1)$ and $k_{0} \in \mathbb{N}$ exist such that for each $k \geq k_{0}$ the following inequality

$$
\left\|u_{k+1}\right\| \leq \beta_{h}\left\|u_{k}\right\|
$$

is satisfied, then the series appearing in (7) is uniformly convergent in interval [a,b]. 
Proof Let $S_{n}$ denote the partial sum of considered series

$$
S_{n}=\sum_{m=0}^{n} u_{m}(x) .
$$

We intend to show that sequence $\left\{S_{n}\right\}$ is the Cauchy sequence. For this purpose we begin by estimating the following norm

$$
\left\|S_{n}-S_{n-1}\right\|=\left\|u_{n}\right\| \leq \beta\left\|u_{n-1}\right\| \leq \ldots \leq \beta^{n-k_{0}}\left\|u_{k_{0}}\right\| .
$$

Now for any $n, k \in \mathbb{N}, n \geq k \geq k_{0}$, we have

$$
\begin{aligned}
& \left\|S_{n}-S_{k}\right\| \leq\left\|S_{n}-S_{n-1}\right\|+\ldots+\left\|S_{k+1}-S_{k}\right\| \\
& \quad \leq \beta^{n-k_{0}}\left\|u_{k_{0}}\right\|+\ldots+\beta^{k+1-k_{0}}\left\|u_{k_{0}}\right\| \\
& =\beta^{k+1-k_{0}}\left(\beta^{n-k-1}+\ldots+\beta+1\right)\left\|u_{k_{0}}\right\|=\beta^{k+1-k_{0}} \frac{1-\beta^{n-k}}{1-\beta}\left\|u_{k_{0}}\right\| .
\end{aligned}
$$

Since $\beta \in(0,1)$, therefore it implies that sequence $\left\{S_{n}\right\}$ is the Cauchy sequence. By using the completeness of space $\mathbb{R}$ we can deduce that this sequence is convergent, which implies convergence of the discussed series.

Remark 2 The above theorem can be generalized in the following way.

If parameter $h$ is selected in such a way that constant $k_{0} \in \mathbb{N}$ exists such that for each $k \geq k_{0}$ there exists $\beta_{h, k} \in(0,1)$ satisfying conditions

$$
\prod_{k=k_{0}}^{\infty} \beta_{h, k}=0 \quad \text { and } \quad\left\|u_{k+1}\right\| \leq \beta_{h, k}\left\|u_{k}\right\|,
$$

then the series appearing in (7) is uniformly convergent in interval $[a, b]$.

Next theorem concerns the estimation of error of the approximate solution $\widehat{u}_{n}$.

Theorem 4 If assumptions of Theorem 3 are satisfied and additionally if $n \in \mathbb{N}$ and $n \geq k_{0}$, then we get the following estimation of error of the approximate solution

$$
\left\|u-\widehat{u}_{n}\right\| \leq \frac{\beta_{h}^{n+1-k_{0}}}{1-\beta_{h}}\left\|u_{k_{0}}\right\| .
$$

Proof Let $n \in \mathbb{N}$ and $n \geq k_{0}$. Thus we obtain

$$
\begin{gathered}
\left\|u-\widehat{u}_{n}\right\|=\sup _{x \in[a, b]}\left|u(x)-\sum_{m=0}^{n} u_{m}(x)\right|=\sup _{x \in[a, b]}\left|\sum_{m=n+1}^{\infty} u_{m}(x)\right| \\
\leq \sup _{x \in[a, b]}\left(\sum_{m=n+1}^{\infty}\left|u_{m}(x)\right|\right) \leq \sum_{m=n+1}^{\infty}\left(\sup _{x \in[a, b]}\left|u_{m}(x)\right|\right)=\sum_{m=n+1}^{\infty}\left\|u_{m}\right\| \\
\leq \sum_{m=n+1}^{\infty} \beta_{h}^{m-k_{0}}\left\|u_{k_{0}}\right\|=\frac{\beta_{h}^{n+1-k_{0}}}{1-\beta_{h}}\left\|u_{k_{0}}\right\| .
\end{gathered}
$$


Remark 3 In particular case when $k_{0}=0$ the formula for estimation of error of the approximate solution takes the form

$$
\left\|u-\widehat{u}_{n}\right\| \leq \frac{\beta_{h}^{n+1}}{1-\beta_{h}}\left\|u_{0}\right\| .
$$

Generalization from Remark 2 can be also applied for Theorem 4.

\section{Linear integral equation}

Let us proceed to consideration of the case when operator $R: C[a, b] \rightarrow C[a, b]$ is a bounded linear operator. Thus we assume $\|R\|<\infty$. In this case operator $R_{m}$ (see (10)) has the form

$$
\begin{aligned}
R_{m}\left(\bar{u}_{m-1}, x\right) & =\frac{1}{(m-1) !}\left(\frac{\partial^{m-1}}{\partial p^{m-1}} N\left(\sum_{i=0}^{\infty} u_{i}(x) p^{i}\right)\right)_{p=0} \\
& =\frac{1}{(m-1) !} \frac{\partial^{m-1}}{\partial p^{m-1}}\left[\sum_{i=1}^{\infty} u_{i}(x) p^{i}-F(x)-\int_{f(x)}^{g(x)} K(x, t) R\left(\sum_{i=1}^{\infty} u_{i}(t) p^{i}\right) d t\right]_{p=0} \\
& =\frac{1}{(m-1) !} \frac{\partial^{m-1}}{\partial p^{m-1}}\left[\sum_{i=1}^{\infty} u_{i}(x) p^{i}-F(x)-\sum_{i=1}^{\infty} \int_{f(x)}^{g(x)} K(x, t) R\left(u_{i}(t)\right) p^{i} d t\right]_{p=0} \\
& =\frac{1}{(m-1) !}\left((m-1) ! u_{m-1}(x)-\left(1-\chi_{m}\right) F(x)\right. \\
& \left.-\int_{f(x)}^{g(x)} K(x, t)(m-1) ! R\left(u_{m-1}(t)\right) d t\right) \\
& =u_{m-1}(x)-\frac{1-\chi_{m}}{(m-1) !} F(x)-\int_{f(x)}^{g(x)} K(x, t) R\left(u_{m-1}(t)\right) d t .
\end{aligned}
$$

By using the above relation and formula (16) we receive the following formulas for functions $u_{m}$ :

$$
u_{1}(x)=h\left(u_{0}(x)-F(x)-\int_{f(x)}^{g(x)} K(x, t) R\left(u_{0}(t)\right) d t\right),
$$

and for $m \geq 2$ :

$$
u_{m}(x)=(1+h) u_{m-1}(x)-h \int_{f(x)}^{g(x)} K(x, t) R\left(u_{m-1}(t)\right) d t .
$$

In case of the linear integral equation Theorem 1 remains true if we take $s=\|R\|$. Whereas in Theorem 2 we do not have to assume that $R$ is the contraction mapping. Respective theorems can be then formulated in the following way.

Theorem 5 If $R$ is the bounded linear operator and the condition given below is fulfilled

$$
\|R\|\|K\|(b-a)<1,
$$

then (14) possesses at most one solution. 
Theorem 6 Let $R$ be the bounded linear operator and let functions $u_{m}, m \geq 1$, be defined by relations (23) and (24). Then, if series in (7) is convergent, the sum of this series is the solution of (14).

Proofs of these two above theorems run similarly as the proofs of Theorems 1 and 2.

Theorem corresponding to Theorem 3 can be, in case of linear equation, formulated in the following way.

Theorem 7 If inequality (25) is satisfied then the series appearing in (7) is uniformly convergent in interval $[a, b]$.

Proof Let $u_{0}$ be the function of class $C[a, b]$. We intend to find the boundaries for function $u_{m}$ in interval $[a, b]$ :

$$
\begin{aligned}
\left|u_{1}(x)\right| & =\left|h\left(u_{0}(x)-F(x)-\int_{f(x)}^{g(x)} K(x, t) R\left(u_{0}(t)\right) d t\right)\right| \\
& \leqslant|h|\left(\left|u_{0}(x)\right|+|F(x)|+\int_{f(x)}^{g(x)}|K(x, t)|\left|R\left(u_{0}(t)\right)\right| d t\right) \\
& \leqslant|h|\left(\|F\|+\left\|u_{0}\right\|+\|K\|\|R\|(b-a)\left\|u_{0}\right\|\right), \\
\left|u_{2}(x)\right| & =\left|(1+h) u_{1}(x)-h \int_{f(x)}^{g(x)} K(x, t) R\left(u_{1}(t)\right) d t\right| \\
& \leqslant(|1+h|+|h|\|K\|\|R\|(b-a))\left\|u_{1}\right\|=\beta_{h}\left\|u_{1}\right\|,
\end{aligned}
$$

where

$$
\beta_{h}:=|1+h|+|h|\|K\|\|R\|(b-a) .
$$

By using the above result one can easily prove by induction that for $m \geqslant 1$ we have

$$
\left\|u_{m}\right\| \leqslant \beta_{h}^{m-1}\left\|u_{1}\right\| \text {. }
$$

In this way, for the considered series (7) we get

$$
\sum_{m=0}^{\infty} u_{m}(x) \leqslant \sum_{m=0}^{\infty}\left|u_{m}(x)\right| \leqslant\left\|u_{0}\right\|+\left\|u_{1}\right\| \sum_{m=1}^{\infty} \beta_{h}^{m-1},
$$

Last series in the above estimation is the geometrical series with quotient $\beta_{h}$. Therefore, if $\beta_{h}<1$ (we have certainly $\beta_{h}>0$ ) then, by virtue of the comparison test, the discussed series is uniformly convergent in interval $[a, b]$.

Let us answer the question whether parameter $h$ can be selected such that $\beta_{h}<1$, it means such that

$$
|1+h|+|h|\|K\|\|R\|(b-a)<1 .
$$

Last inequality is equivalent to condition ( since $h \neq 0$ ):

$$
\|K\|\|R\|(b-a)<\frac{1-|1+h|}{|h|} .
$$


One can easily notice that

$$
\frac{1-|1+h|}{|h|}= \begin{cases}-1-\frac{2}{h} & \text { for } h<-1, \\ 1 & \text { for } h \in[-1,0), \\ -1 & \text { for } h>0 .\end{cases}
$$

It implies that if condition (25) is fulfilled then we are able to choose the value of parameter $h$ such that inequality (26) will be satisfied (for this aim it is enough to take any $h \in[-1,0))$, which means that $\beta_{h}<1$.

By using the estimation evaluated in the last proof we can prove the following theorem.

Theorem 8 If inequality (25) is fulfilled and $n \in \mathbb{N}$ then we get the following estimation of error of the approximate solution

$$
\left\|u-\widehat{u}_{n}\right\| \leqslant \frac{\beta_{h}^{n}}{1-\beta_{h}}\left\|u_{1}\right\|,
$$

where $\beta_{h}=|1+h|+|h|\|K\|\|R\|(b-a)$.

Remark 4 Let us notice that if we take $h=-1$ and $u_{0}(x)=0$ or $u_{0}(x)=F(x)$ then the investigated homotopy analysis method is equivalent to the method of successive approximation as well as to the Adomian decomposition method (in case $u_{0}(x)=0$ after removing the first term which is identically equal to zero - see also $[2,53])$. It concerns the case of nonlinear equations as well. Whereas for $h=-1$ the method is identical with the homotopy perturbation method (see [23-25]).

Remark 5 In literature (see for example [29, 33]) some other formulation for homotopy operator can be found, which is

$$
\mathcal{H}(\Phi, p) \equiv(1-p) L\left(\Phi(x ; p)-u_{0}(x)\right)-p h H(x) N(\Phi(x ; p)),
$$

where $H$ is the auxiliary function. In this case as well, all the results evaluated in this paper (for nonlinear and linear equations too) hold true. Only the formula for constant $\beta_{h}$ will change.

\section{Examples}

Example 1 Let us use the investigated method for solving equation of the form

$$
u(x)-\frac{1}{3} \int_{0}^{1}(x-t)(u(t))^{2} d t=\frac{8}{9} x+\frac{1}{12},
$$

for $x \in[0,1]$. Solution of the above equation is given by function $u_{e}(x)=x$. In considered equation we can take $K(x, t)=x-t$ and $R(u)=u^{2} / 3$. Then we have

$$
\|K\|=1, \quad s=\frac{2}{3} .
$$


Hence

$$
s\|K\|(b-a)=\frac{2}{3},
$$

which means that the discussed equation possesses at most one solution and if the constructed series is convergent, then its sum is the solution of this equation.

By taking the initial approximation as zero: $u_{0}(x)=0$, we get successively

$$
\begin{aligned}
u_{1}(x)= & -\frac{1}{36} h(3+32 x), \\
u_{2}(x)=- & \frac{1}{36} h(1+h)(3+32 x), \\
u_{3}(x)=- & \frac{1}{23328} h\left(648(3+32 x)+1296 h(3+32 x)+h^{2}(-3+23414 x)\right), \\
u_{4}(x)=- & \frac{1}{7776} h(1+h)(216(3+32 x) \\
& \left.+432 h(3+32 x)+h^{2}(-1299+9590 x)\right), \\
u_{5}(x)=- & \frac{1}{7558272} h(209952(3+32 x)+839808 h(3+32 x) \\
& +3888 h^{3}(-1299+9590 x)+1944 h^{2}(-3+23414 x) \\
& \left.+h^{4}(-3157593+11898032 x)\right),
\end{aligned}
$$

In Fig. 1 the plots of logarithm of squared residual $E_{n}$ for $n=3,5,7$ are presented. In this case the numerically determined, by minimizing the squared residual of governing equations, optimal value of the convergence control parameter was equal to $-1(h=-1)$. Figure 2 presents the $h$-curve of $u^{\prime}(0)$.

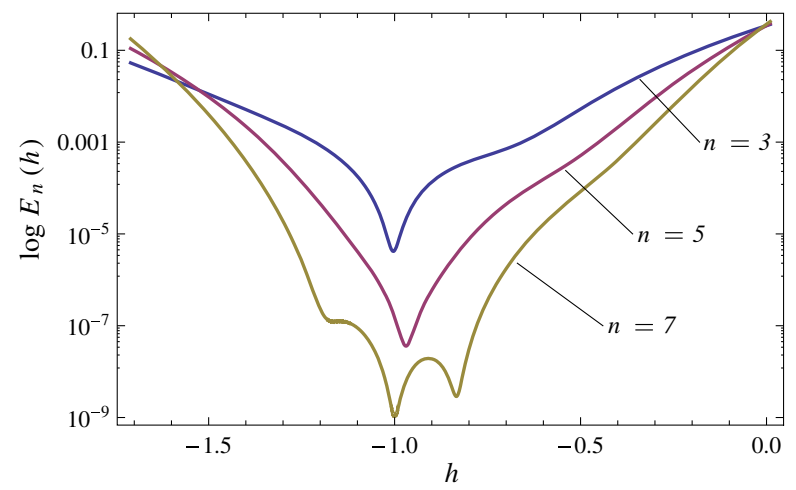

Fig. 1 Logarithm of squared residual $E_{n}$ for $n=3,5,7$ 


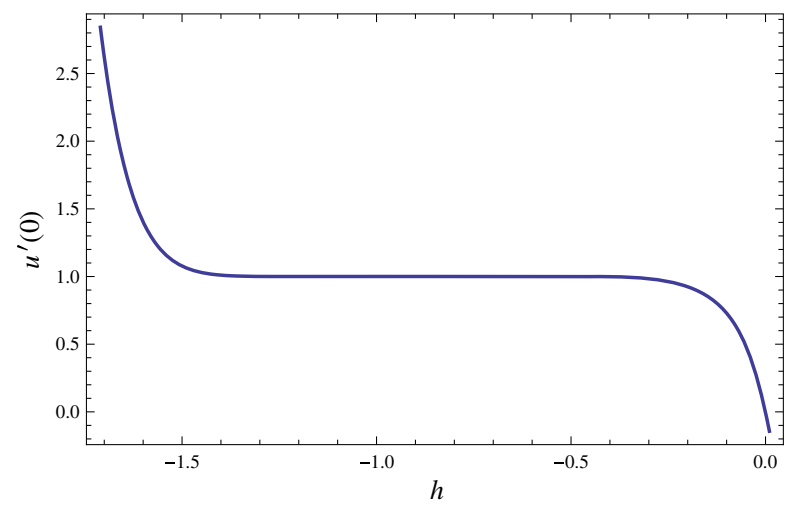

Fig. 2 The $h$-curve of $u^{\prime}(0)$

By calculating the partial sums of series in (7) for $h=-1$, which means by determining the successive approximate solutions $\widehat{u}_{n}$, we obtain

$$
\begin{aligned}
\widehat{u}_{1}(x) & =0.0833333+0.888889 x, \\
\widehat{u}_{3}(x) & =-1.28601 \cdot 10^{-4}+1.00369 x, \\
\widehat{u}_{5}(x) & =-4.5685 \cdot 10^{-4}+1.00019 x, \\
\widehat{u}_{7}(x) & =7.06342 \cdot 10^{-5}+0.999873 x, \\
\widehat{u}_{9}(x) & =5.05985 \cdot 10^{-6}+x, \\
\widehat{u}_{11}(x) & =-1.57935 \cdot 10^{-6}+x, \\
\widehat{u}_{13}(x) & =6.01773 \cdot 10^{-8}+x, \\
\widehat{u}_{15}(x) & =3.35277 \cdot 10^{-8}+x,
\end{aligned}
$$

where $x \in[0,1]$. In considered equation for $h=-1$ the equality $\widehat{u}_{2 n-1}(x) \equiv \widehat{u}_{2 n}(x)$ holds.

Table 1 compiles the percentage relative errors of the exact solution reconstruction for various values of the convergence control parameter $h$. As revealed by the above results, together with increase of the components number in sum (11) the errors quickly decrease. The fastest error decrease can be observed for optimal value $h=-1$. For this value the approximate solution $\widehat{u}_{5}$ provides the approximation of the sought function with the error not higher than 6.3764 . $10^{-2} \%$, while the approximate solution $\widehat{u}_{15}$ gives the error not higher than 4.0685 . $10^{-6} \%$. Whereas, by moving further away from this value, the errors decrease slower.

Differences $\left|u_{e}(x)-\widehat{u}_{n}(x)\right|$ for $n=5$ and $n=15$ are displayed in Fig. 3. Obtained results indicate that the method is very rapidly convergent and calculation of only few first terms of the series ensures very good approximation of the exact solution. 
Table 1 Values of the percentage relative errors in reconstruction of the exact solution

\begin{tabular}{lllllll}
\hline$n$ & $h=-1.25$ & $h=-1.1$ & $h=-1$ & $h=-0.9$ & $h=-0.75$ & $h=-0.5$ \\
\hline 1 & 28.217 & 13.997 & 7.349 & 10.897 & 24.562 & 49.437 \\
2 & 8.382 & 7.155 & 7.349 & 7.155 & 8.382 & 24.562 \\
3 & 6.853 & 2.399 & 0.350 & 2.011 & 4.104 & 12.632 \\
4 & 3.691 & 0.633 & 0.350 & 0.538 & 1.866 & 6.937 \\
5 & 1.960 & 0.336 & $6.376 \cdot 10^{-2}$ & 0.126 & 0.770 & 4.092 \\
6 & 0.948 & $3.643 \cdot 10^{-2}$ & $6.376 \cdot 10^{-2}$ & $2.733 \cdot 10^{-2}$ & 0.286 & 2.531 \\
7 & 0.293 & $5.240 \cdot 10^{-2}$ & $6.458 \cdot 10^{-3}$ & $2.289 \cdot 10^{-2}$ & $8.986 \cdot 10^{-2}$ & 1.592 \\
8 & 0.115 & $2.194 \cdot 10^{-2}$ & $6.458 \cdot 10^{-3}$ & $9.243 \cdot 10^{-3}$ & $1.926 \cdot 10^{-2}$ & 0.997 \\
9 & 0.140 & $1.082 \cdot 10^{-2}$ & $1.097 \cdot 10^{-3}$ & $3.426 \cdot 10^{-3}$ & $1.544 \cdot 10^{-3}$ & 0.616 \\
10 & 0.113 & $4.319 \cdot 10^{-3}$ & $1.097 \cdot 10^{-3}$ & $1.406 \cdot 10^{-3}$ & $4.313 \cdot 10^{-3}$ & 0.373 \\
11 & $7.162 \cdot 10^{-2}$ & $2.661 \cdot 10^{-3}$ & $1.472 \cdot 10^{-4}$ & $6.982 \cdot 10^{-4}$ & $3.141 \cdot 10^{-3}$ & 0.221 \\
12 & $4.285 \cdot 10^{-2}$ & $1.424 \cdot 10^{-3}$ & $1.472 \cdot 10^{-4}$ & $2.721 \cdot 10^{-4}$ & $1.719 \cdot 10^{-3}$ & 0.128 \\
13 & $3.094 \cdot 10^{-2}$ & $4.876 \cdot 10^{-4}$ & $1.858 \cdot 10^{-5}$ & $7.296 \cdot 10^{-5}$ & $8.493 \cdot 10^{-4}$ & $7.184 \cdot 10^{-2}$ \\
14 & $2.319 \cdot 10^{-2}$ & $1.745 \cdot 10^{-4}$ & $1.858 \cdot 10^{-5}$ & $2.246 \cdot 10^{-5}$ & $4.242 \cdot 10^{-4}$ & $3.914 \cdot 10^{-2}$ \\
15 & $1.471 \cdot 10^{-2}$ & $1.150 \cdot 10^{-4}$ & $4.069 \cdot 10^{-6}$ & $1.474 \cdot 10^{-5}$ & $2.208 \cdot 10^{-4}$ & $2.055 \cdot 10^{-2}$ \\
\hline
\end{tabular}

Example 2 In the next example we deal with equation which may be practically applied for calculating the charge in supply circuit of flash lamps used in cameras [15, 24]. Supply circuit of flash lamps may be pictured as a simple electrical circuit consisting of source and series connected ideal switch, resistor and capacitor. For modeling the charging or discharging process the first convolution integral may be used. In this example we consider the equation representing the charge referred to some value which gives the input signal for main controller to stop, for example, the charging process of the capacitor or discharging the capacitor by connected flash lamp. Part of this equation, connected with second integral, may represent the

$\mathbf{a}$

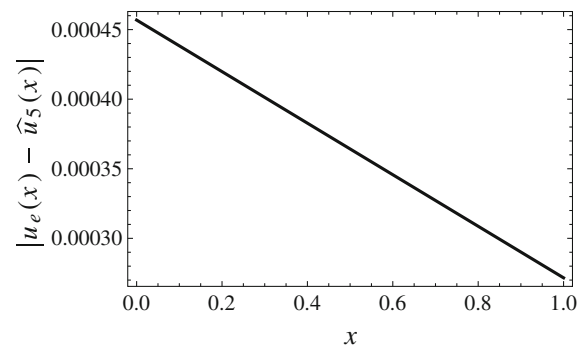

b

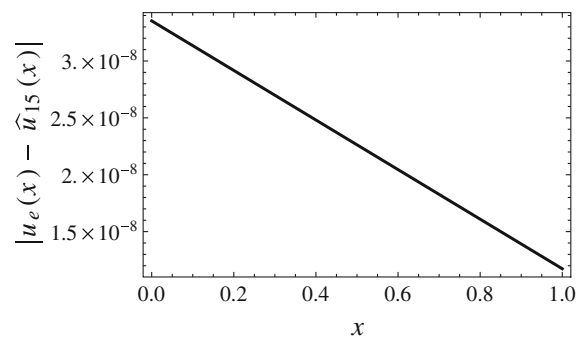

Fig. 3 Distribution of error of the exact solution approximation for $n=5(a)$ and $n=15(b)$ 
simplified measurement circuit (with characteristic described by integral function) calculating the charge collected on capacitor in some time interval.

Thus we seek for the solution of integral equation of the form

$$
u(x)-\int_{0}^{x}(x-t)\left(\int_{0}^{t} v(\tau) u(t-\tau) d \tau\right) d t=F(x)
$$

for $x \in[0,1]$ where (see Fig. 4):

$$
v(t)= \begin{cases}\frac{1}{2}, & t \in\left[0, \frac{1}{4}\right) \cup\left[\frac{3}{4}, 1\right], \\ 1, & t \in\left[\frac{1}{4}, \frac{3}{4}\right)\end{cases}
$$

and

$$
F(x)= \begin{cases}\frac{1}{24}\left(48 x-x^{4}\right), & x \in\left[0, \frac{1}{4}\right], \\ \frac{1}{6144}\left(-1+12304 x-96 x^{2}+256 x^{3}-512 x^{4}\right), & x \in\left(\frac{1}{4}, \frac{1}{2}\right], \\ \frac{1}{6144}\left(12319-12528 x+672 x^{2}-768 x^{3}\right), & x \in\left(\frac{1}{2}, \frac{3}{4}\right], \\ \frac{1}{3072}\left(6281-6912 x+1632 x^{2}-1536 x^{3}+384 x^{4}\right), & x \in\left(\frac{3}{4}, 1\right] .\end{cases}
$$

Plot of function $F$ looks illusively like some polyline, but certainly it is not (see formula above).

Exact solution of the above equation has the form (see Fig. 5):

$$
u_{e}(x)= \begin{cases}2 x, & x \in\left[0, \frac{1}{2}\right), \\ 2(1-x), & x \in\left[\frac{1}{2}, 1\right] .\end{cases}
$$

In Fig. 6 the plots of logarithm of squared residual $E_{n}$ for $n=3,4,5$ are shown. Numerically determined, by minimizing the squared residual of governing equations, optimal value of the convergence control parameter was $h=-1$. Figure 7 presents the $h$-curve of $u^{\prime}(0)$.

a

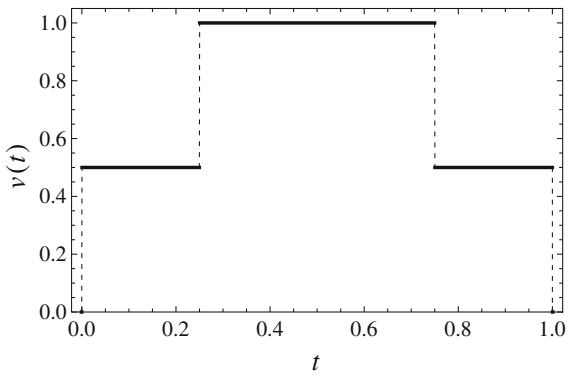

b

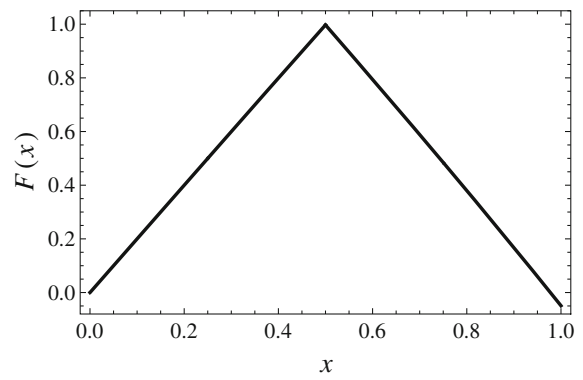

Fig. 4 Functions $v$ and $F$ 


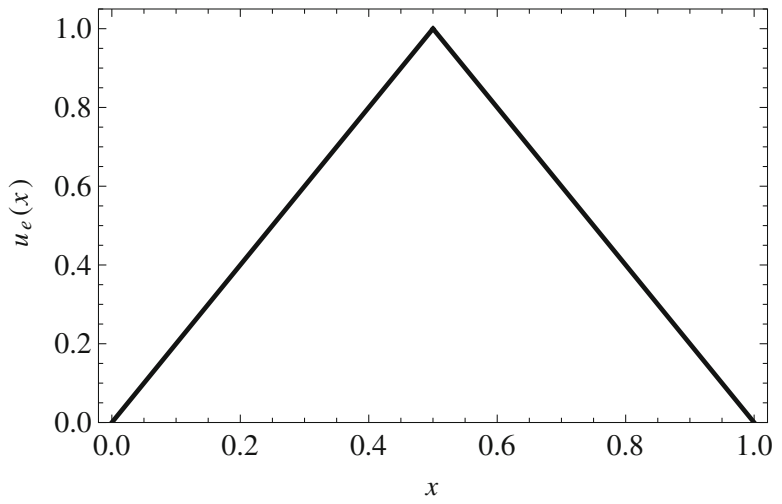

Fig. 5 Exact solution of (29)

By taking the zero initial approximation $u_{0}(x)=0$ and the optimal value of convergence control parameter we receive successively

$$
\begin{aligned}
& u_{1}(x)= \\
& u_{2}(x)= \begin{cases}\frac{1}{10080} x^{4}\left(420-x^{3}\right), & x \in\left[0, \frac{1}{4}\right], \\
\frac{1}{82575360}\left(13441-215068 x+1290576 x^{2}-3442880 x^{3}\right. & \\
\left.+6890240 x^{4}-21504 x^{5}+28672 x^{6}-24576 x^{7}\right), & x \in\left(\frac{1}{4}, \frac{1}{2}\right], \\
\frac{1}{82575360}\left(-416703+3226468 x-9036720 x^{2}\right. & \\
\left.+10337600 x^{3}-26880 x^{4}+21504 x^{5}-16384 x^{7}\right), & x \in\left(\frac{1}{2}, \frac{3}{4}\right], \\
\frac{1}{10321920}\left(-461148+2588243 x-5514768 x^{2}\right. & \\
+5230960 x^{3}-1384320 x^{4}+75264 x^{5}-32256 x^{6} & \\
\left.+4096 x^{7}\right), & x \in\left(\frac{3}{4}, 1\right] .\end{cases}
\end{aligned}
$$

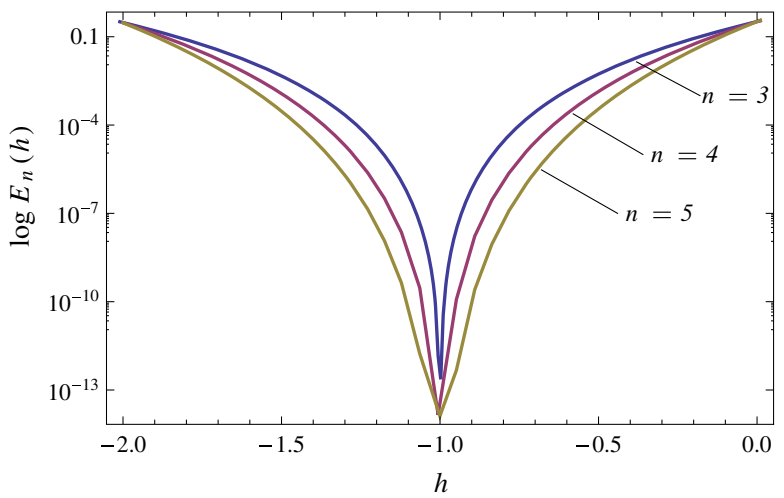

Fig. 6 Logarithm of squared residual $E_{n}$ for $n=3,4,5$ 


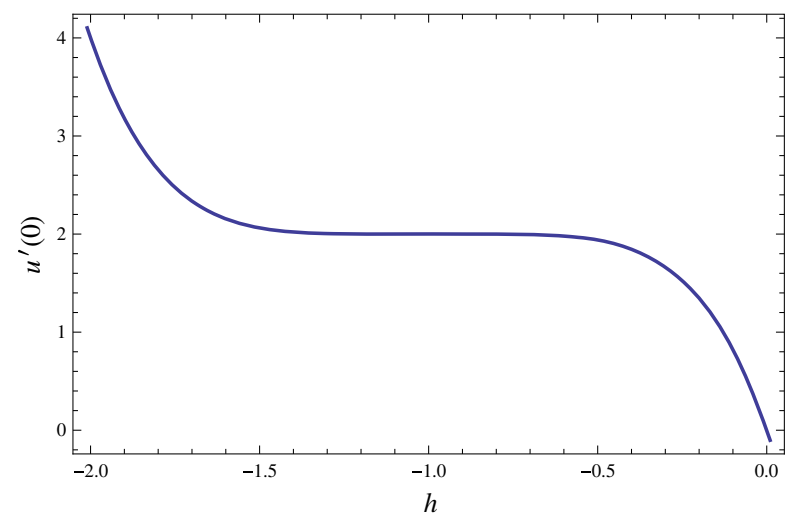

Fig. 7 The $h$-curve of $u^{\prime}(0)$

Table 2 displays the absolute errors $(\Delta)$ and the percentage relative errors $(\delta)$ with which the approximate solution $\widehat{u}_{n}$ approaches the exact solution $u_{e}$. Differences $\left|u_{e}(x)-\widehat{u}_{n}(x)\right|$ for $n=2,4$ are displayed in Fig. 8 .

There are also presented in table the estimations of error of the successive approximate solutions resulting from relation (27). In considered example we have $\|K\|=1$, $\|F\|=1$ and $\|R\|=3 / 4$. Thereby for the optimal value of convergence control parameter $h=-1$ we obtain $\beta_{-1}=3 / 4,\left\|u_{1}\right\|=\|F\|=1$ and

$$
\left\|u-\widehat{u}_{n}\right\| \leqslant \frac{\beta_{-1}^{n}}{1-\beta_{-1}}\left\|u_{1}\right\|=4\left(\frac{3}{4}\right)^{n} \text {. }
$$

Presented data imply that the real errors of approximate solution are significantly smaller than the estimations following from relation (27).

As indicated by the example, with the properly chosen value of convergence control parameter $h$, if it is impossible to predict a general form of function $u_{m}$ or calculate the sum of series in (7), it is sufficient to use the sum of several first functions $u_{m}$ to obtain a very good approximation of sought solution.

Table 2 Errors of the exact solution approximation ( $\Delta-$ absolute error, $\delta-$ percentage relative error) and estimation of the error resulting from relation (27)

\begin{tabular}{llll}
\hline$n$ & $\Delta$ & $\delta[\%]$ & $(27)$ \\
\hline 1 & $1.644410^{-2}$ & 2.8481 & 3.0000 \\
2 & $3.134310^{-5}$ & $5.428810^{-3}$ & 2.2500 \\
3 & $1.722110^{-8}$ & $2.982810^{-6}$ & 1.6875 \\
4 & $4.179110^{-12}$ & $7.238510^{-10}$ & 1.2656 \\
5 & $5.423710^{-16}$ & $9.394210^{-14}$ & 0.9492 \\
6 & $4.203110^{-20}$ & $7.280010^{-18}$ & 0.7119 \\
7 & $2.094510^{-24}$ & $3.627710^{-22}$ & 0.5339 \\
8 & $7.089210^{-29}$ & $1.227910^{-26}$ & 0.4005 \\
\hline
\end{tabular}


a

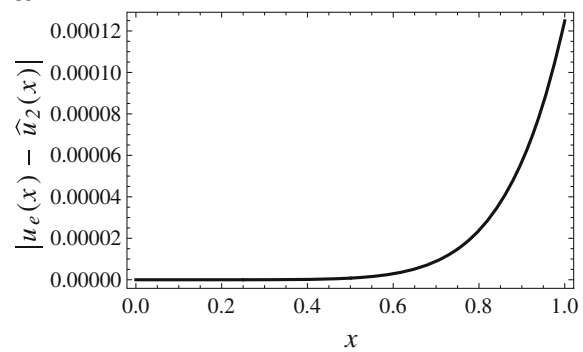

b

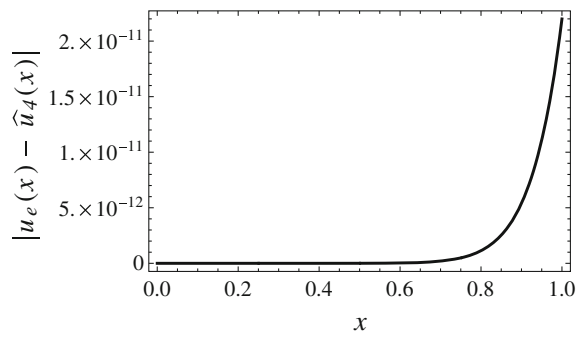

Fig. 8 Distribution of error of the exact solution approximation for $n=2(a)$ and $n=4(b)$

Example 3 Next example shows that the discussed method may be effectively applied for solving linear equations not satisfying inequality (25). Let us consider equation of the form [47]:

$$
u(x)-\int_{0}^{\pi} \cos x \cos t u(t) d t=\left(1-\frac{\pi}{2}\right) \cos x+\sin x,
$$

for $x \in[0, \pi]$. Solution of the above equation is given by function $u_{e}(x)=\cos x+$ $\sin x$. In this case we have

$$
\|K\|=1, \quad\|R\|=1 .
$$

Hence

$$
\|R\|\|K\|(b-a)=\pi>1 .
$$

By taking the initial approximation of the form

$$
u_{0}(x)=\left(1-\frac{\pi}{2}\right) \cos x+\sin x
$$

we receive successively

$$
\begin{aligned}
& u_{1}(x)=\frac{1}{4} h(\pi-2) \pi \cos x, \\
& u_{2}(x)=-\frac{1}{8}(h(\pi-2)-2) h(\pi-2) \pi \cos x, \\
& u_{3}(x)=\frac{1}{16}(h(\pi-2)-2)^{2} h(\pi-2) \pi \cos x, \\
& u_{4}(x)=-\frac{1}{32}(h(\pi-2)-2)^{3} h(\pi-2) \pi \cos x, \\
& u_{5}(x)=\frac{1}{64}(h(\pi-2)-2)^{4} h(\pi-2) \pi \cos x,
\end{aligned}
$$

One can easily prove by induction that for any $m \in \mathbb{N}$ we get

$$
u_{m}(x)=\left(-\frac{1}{2}\right)^{m+1}(h(\pi-2)-2)^{m-1} h(\pi-2) \pi \cos x .
$$


From this we have

$$
\begin{aligned}
u(x)= & u_{0}(x)+\sum_{m=1}^{\infty} u_{m}(x)=\left(1-\frac{\pi}{2}\right) \cos x+\sin x \\
& +h(\pi-2) \pi \cos x \sum_{m=1}^{\infty}\left(\left(-\frac{1}{2}\right)^{m+1}(h(\pi-2)-2)^{m-1}\right) \\
= & \left(1-\frac{\pi}{2}\right) \cos x+\sin x+h(\pi-2) \pi \cos x \frac{\frac{1}{4}}{1-\left(-\frac{1}{2}\right)(h(\pi-2)-2)} \\
= & \left(1-\frac{\pi}{2}\right) \cos x+\sin x+h(\pi-2) \pi \cos x \frac{1}{2 h(\pi-2)} \\
= & \cos x+\sin x,
\end{aligned}
$$

if only the condition of convergence of the geometrical sequence is satisfied, which in this case takes the form

$$
\left|\left(-\frac{1}{2}\right)(h(\pi-2)-2)\right|<1 .
$$

The above inequality is satisfied if

$$
h \in\left(0, \frac{4}{\pi-2}\right) .
$$

Certainly the above interval determines the effective region of the convergence control parameter $\mathbf{R}_{h}=(0,4 /(\pi-2))$. It means that by taking any value from the above interval we obtain the exact solution of the above equation.

In considered example we can determine in analytical way the squared residual of governing equations

$$
E_{n}(h)=\frac{1}{512} 4^{2-n} \pi^{3}(\pi-2)^{2}(h(\pi-2)-2)^{2 n}, \quad n \in \mathbb{N} .
$$

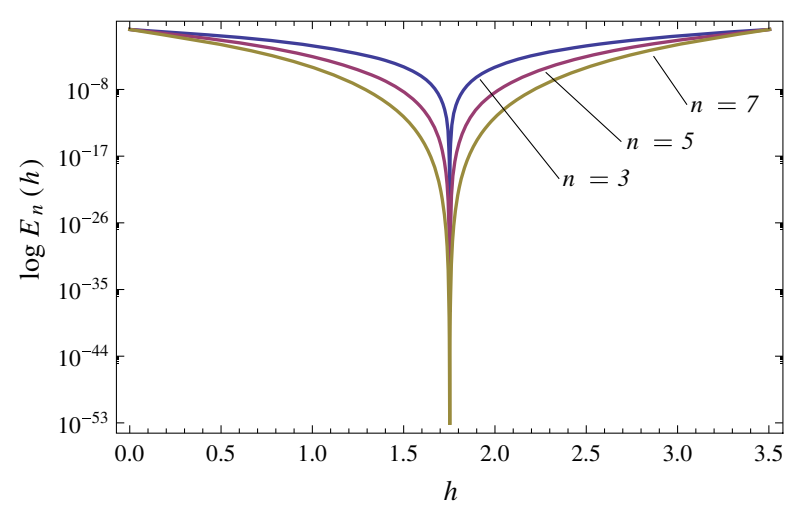

Fig. 9 Logarithm of squared residual $E_{n}$ for $n=3,5,7$ 


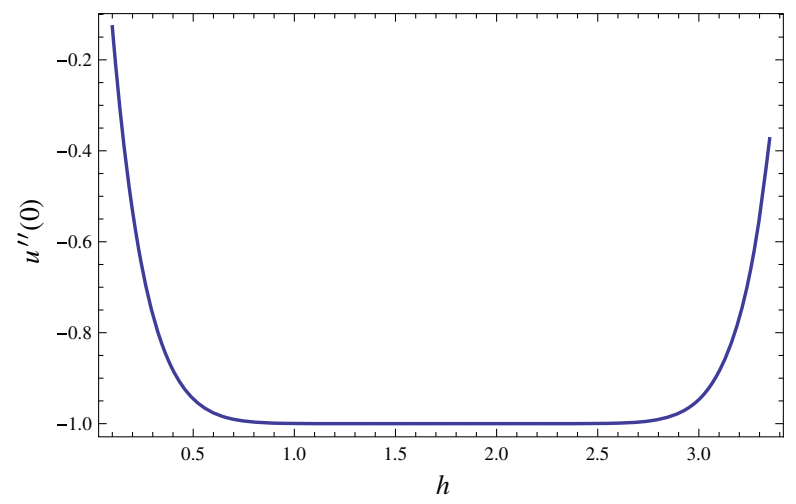

Fig. 10 The $h$-curve of $u^{\prime \prime}(0)$

Logarithms of squared residual $E_{n}$, for $n=3,5,7$, are presented in Fig. 9. Whereas, Fig. 10 presents the $h$-curve of $u^{\prime \prime}(0)$. One can easily verify that for any $n \in \mathbb{N}$ function $E_{n}$ takes minimum for

$$
h=\frac{2}{\pi-2} .
$$

It means that the optimal value of convergence control parameter $h$ is equal to $2 /(\pi-2)$.

\section{Conclusion}

In this paper the homotopy analysis method has been successfully applied for solving the nonlinear and linear integral equations of the second kind. The homotopy analysis method consists in formulating the series, elements of which are iteratively determined. We prove that if this series is convergent then its sum is a solution of considered equation. This series contains the convergence control parameter $h$. Appropriate selection of this parameter influence the region of convergence of the series and the convergence rate. In many case we are able to determine analytically the sum of obtained series and, in this way, to compute the exact solution of considered equation. Whereas in cases when analytical determination of sum of the series is impossible, we can use its initial terms for creating the approximate solution. In most of cases, with regard to the rapid convergence of considered series, only few initial terms ensures a very small error of the exact solution approximation. Condition ensuring the convergence of obtained series is presented in the paper and the error of approximate solution received by taking the partial sum of the series is estimated as well. Presented examples show that investigated method is effective in solving the equations of considered kind. Proved Theorems 3 and 7 define the sufficient conditions for convergence of the series constructed in the course of applying the homotopy analysis method. Additionally the third example shows that the method can be used as well for equations not satisfying the conditions given in the above theorems. 
Acknowledgments The Authors would like to thank the Referees and the Editor Shijun Liao for their constructive comments and suggestions.

Open Access This article is distributed under the terms of the Creative Commons Attribution License which permits any use, distribution, and reproduction in any medium, provided the original author(s) and the source are credited.

\section{References}

1. Abbasbandy, S.: Homotopy analysis method for heat radiation equations. Int. Comm. Heat Mass Transf. 34, 380-387 (2006)

2. Abbasbandy, S., Shivanian, E.: A new analytical technique to solve Fredholm's integral equations. Numer. Algor. 56, 27-43 (2011)

3. Abbasbandy, S., Shivanian, E., Vajravelu, K.: Mathematical properties of h-curve in the frame work of the homotopy analysis method. Commun. Nonlinear Sci. Numer. Simulat. 16, 4268-4275 (2011)

4. Abdulaziz, O., Bataineh, A., Hashim, I.: On convergence of homotopy analysis method and its modification for fractional modified KdV equations. J. Appl. Math. Comput. 33, 61-81 (2010)

5. Afroozi, G., Vahidi, J., Saeidy, M.: Solving a class of two-dimensional linear and nonlinear Volterra integral equations by means of the homotopy analysis method. Int. J. Nonlinear Sci. 9, 213-219 (2010)

6. Akyildiz, F., Vajravelu, K.: Magnetohydrodynamic flow of a viscoelastic fluid. Phys. Lett. A 372, 3380-3384 (2008)

7. Akyildiz, F., Vajravelu, K., Mohapatra, R., Sweet, E., Van Gorder, R.A.: Implicit differential equation arising in the steady flow of a Sisko fluid. Appl. Math. Comput. 210, 189-196 (2009)

8. Allahviranloo, T., Ghanbari, M.: Discrete homotopy analysis method for the nonlinear Fredholm integral equations. Ain Shams Eng. J. 2, 133-140 (2011)

9. Araghi, M., Behzadi, S.: Numerical solution of nonlinear Volterra-Fredholm integro-differential equations using homotopy analysis method. J. Appl. Math. Comput. 37, 1-12 (2011)

10. Awawdeh, F., Adawi, A., Al-Shara', S.: A numerical method for solving nonlinear integral equations. Int. Math. Forum 4(17), 805-817 (2009)

11. Baxter, M., Van Gorder, R.A.: Exact and analytic solutions of the Ernst equation governing axially symmetric stationary vacuum gravitational fields. Phys. Scr. 87, 035005 (2013)

12. Bazrafshan, F., Mahbobi, A., Neyrameh, A., Sousaraie, A., Ebrahimi, M.: Solving two-dimensional integral equations. J. King Saud Univ. (Sci.) 23, 111-114 (2011)

13. Behzadi, S.: Solving fuzzy nonlinear Volterra-Fredholm integral equations by using homotopy analysis and Adomian decomposition methods. J. Fuzzy Set Valued Anal. 2011 (2011). article ID jfsva-00067

14. Behzadi, S.: Application of homotopy analysis method for solving a class of nonlinear VolterraFredholm integro-differential equations. Adv. Dev. Technol. Int. 1, 1-14 (2012)

15. Campo, J.C., Pkrez, M.A., Mezquita, J.M., Sebastian, J.: Circuit-design criteria for improvement of xenon flash-lamp performance (lamp life, light-pulse, narrowness, uniformity of light intensity in a series of flashes). Appl. Power Electron. Conf. Expo., APEC'97, Twelfth Ann. 2, 1057-1061 (1997)

16. Chauhan, D., Agrawal, R., Rastogi, P.: Magnetohydrodynamic slip flow and heat transfer in a porous medium over a stretching cylinder: homotopy analysis method. Numer. Heat Transf. A 62, 136-157 (2012)

17. Cherruault, Y.: Convergence of Adomian's method. Kybernetes 18, 31-38 (1989)

18. Domairry, G., Nadim, N.: Assessment of homotopy analysis method and homotopy perturbation method in non-linear heat transfer equation. Int. Comm. Heat Mass Transf. 35, 93-102 (2008)

19. Fan, T., You, X.: Optimal homotopy analysis method for nonlinear differential equations in the boundary leyer. Numer. Algor. 62, 337-354 (2013)

20. Ghoreishi, M., Ismail, A., Alomari, A.: Comparison between homotopy analysis method and optimal homotopy asymptotic method for nth-order integro-differential equation. Math. Methods Appl. Sci. 34, 1833-1842 (2011) 
21. Ghoreishi, M., Ismail, A., Alomari, A., Bataineh, A.S.: The comparison between homotopy analysis method and optimal homotopy asymptotic method for nonlinear age structured population models. Commun. Nonlinear Sci. Numer. Simulat. 17, 1163-1177 (2012)

22. Hashmi, M., Khan, N., Iqbal, S.: Optimal homotopy asymptotic method foe solving nonlinear fredholm integral equations of second kind. Appl. Math. Comput. 218, 10982-10989 (2012)

23. Hetmaniok, E., Nowak, I., Słota, D., Wituła, R.: A study of the convergence of and error estimation for the homotopy perturbation method for the Volterra-Fredholm integral equations. Appl. Math. Lett. 26, 165-169 (2013)

24. Hetmaniok, E., Słota, D., Trawiński, T., Wituła, R., Wituła, R.: An analytical technique for solving general linear integral equations of the second kind and its application in analysis of flash lamp control circuit Bull. Pol. Acad. Sci. Tech. Sci. in review (2013)

25. Hetmaniok, E., Słota, D., Wituła, R.: Convergence and error estimation of homotopy perturbation method for Fredholm and Volterra integral equations. Appl. Math. Comput. 218, 10717-10725 (2012)

26. Hosseini, K., Daneshian, B., Amanifard, N., Ansari, R.: Homotopy analysis method for a fin with temperature dependent internal heat generation and thermal conductivity. Int. J. Nonlin. Sci. 14, 201$210(2012)$

27. Jafari, H., Firoozjaee, M.: Multistage homotopy analysis method for solving nonlinear integral equations. Appl. Appl. Math. Int. J. 1, 34-45 (2010)

28. Liao, S.: Homotopy analysis method: a new analytic method for nonlinear problems. Appl. Math. Mech. Engl. Ed. 19, 957-962 (1998)

29. Liao, S.: Beyond Perturbation: Introduction to the Homotopy Analysis Method. Chapman and HallCRC Press, Boca Raton (2003)

30. Liao, S.: On the homotopy analysis method for nonlinear problems. Appl. Math. Comput. 147, 499$513(2004)$

31. Liao, S.: Notes on the homotopy analysis method: some definitions and theorems. Commun. Nonlinear Sci. Numer. Simulat. 14, 983-997 (2009)

32. Liao, S.: An optimal homotopy-analysis approach for strongly nonlinear differential equations. Commun. Nonlinear Sci. Numer. Simulat. 15, 2003-2015 (2010)

33. Liao, S.: Homotopy Analysis Method in Nonlinear Differential Equations. Springer/Higher Education Press, Berlin/Beijing (2012)

34. Marinca, V., Herisanu, N.: Nonlinear Dynamical Systems in Engineering. Springer, Berlin (2011)

35. Mesloub, S., Obaida, S.: On the application of the homotopy analysis method for a nonlocal mixed problem with Bessel operator. Appl. Math. Comput. 219, 3477-3485 (2012)

36. Molabahrami, A., Shidfar, A., Ghyasi, A.: An analytical method for solving linear Fredholm fuzzy integral equations of the second kind. Comput. Math. Appl. 61, 2754-2761 (2011)

37. Nik, H., Effati, S., Motsa, S., Shirazian, M.: Spectral homotopy analysis method and its convergence for solving a class of nonlinear optimal control problems. Numer. Algor. (2013). doi:10.1007/s11075-013-9700-4. in print

38. Odibat, Z.: A study on the convergence of homotopy analysis method. Appl. Math. Comput. 217, $782-789$ (2010)

39. Russo, M., Van Gorder, R.A.: Control of error in the homotopy analysis of nonlinear Klein-Gordon initial value problems. Appl. Math. Comput. 219, 6494-6509 (2013)

40. Shayganmanesh, A.: Generalizing homotopy analysis method to solve system of integral equations. J. Math. Ext. 5, 21-30 (2010)

41. Shidfar, A., Babaei, A., Molabahrami, A., Alinejadmofrad, M.: Approximate analytical solutions of the nonlinear reaction-diffusion-convection problems. Math. Comput. Model. 53, 261-268 (2011)

42. Shidfar, A., Molabahrami, A.: Solving a system of integral equations by an analytic method. Math. Comput. Model. 54, 828-835 (2011)

43. Shidfar, A., Molabahrami, A., Babaei, A., Yazdanian, A.: A series solution of the nonlinear Volterra and Fredholm integro-differential equations. Commun. Nonlinear Sci. Numer. Simulat. 15, 205-215 (2010)

44. Turkyilmazoglu, M.: A note on the homotopy analysis method. Appl. Math. Lett. 23, 1226-1230 (2010)

45. Turkyilmazoglu, M.: Convergence of the homotopy analysis method. arXiv:1006.4460v1 (2010)

46. Turkyilmazoglu, M.: Some issues on HPM and HAM: a convergence scheme. Math. Comput. Model. 53, 1929-1936 (2011) 
47. Vahdati, S.: Computational methods for integral equations. Lambert Acad. Publ., Saarbrücken (2012)

48. Vahdati, S., Abbas, Z., Ghasemi, M.: Applicaion of homotopy analysis method to Fredholm and Volterra integral equations. Mathematical Sciences 4, 267-282 (2010)

49. Van Gorder, R.A.: Analytical method for the construction of solutions to the Foppl一von Karman equations governing deflections of a thin flat plate. Int. J. Non-Linear Mech. 47, 1-6 (2012)

50. Van Gorder, R.A.: Control of error in the homotopy analysis of semi-linear elliptic boundary value problems. Numer. Algor. 61, 613-629 (2012)

51. Van Gorder, R.A.: Gaussian waves in the Fitzhugh-Nagumo equation demonstrate one role of the auxiliary function $h(x)$ in the homotopy analysis method. Commun. Nonlinear Sci. Numer. Simulat. 17, 1233-1240 (2012)

52. Van Gorder, R.A., Vajravelu, K.: On the selection of auxiliary functions, operators, and convergence control parameters in the application of the homotopy analysis method to nonlinear differential equations: a general approach. Commun. Nonlinear Sci. Numer. Simulat. 14, 4078-4089 (2009)

53. Vosughi, H., Shivanian, E., Abbasbandy, S.: A new analytical technique to solve Volterra's integral equations. Math. Methods Appl. Sci. 34, 1243-1253 (2011)

54. Xu, H., Cang, J.: Analysis of a time fractional wave-like equation with the homotopy analysis method. Phys. Lett. A 372, 1250-1255 (2008)

55. Yabushita, K., Yamashita, M., Tsuboi, K.: An analytic solution of projectile motion with the quadratic resistance law using the homotopy analysis method. J. Phys. A Math. Theor. 40, 8403-8416 (2007)

56. You, X., Xu, H., Pop, I.: Homotopy analysis of unsteady heat transfer started impulsively from rest along a symmetric wedge. Int. Comm. Heat Mass Transf. 37, 47-51 (2010)

57. Zhang, X., Tang, B., He, Y.: Homotopy analysis method for higher-order fractional integro-differential equations. Comput. Math. Appl. 62, 3194-3203 (2011)

58. Zurigat, M., Momani, S., Odibat, Z., Alawneh, A.: The homotopy analysis method for handling systems of fractional differential equations. Appl. Math. Modelling 34, 24-35 (2010) 